\title{
A SHORT WZ-STYLE PROOF OF ABEL'S IDENTITY
}

\author{
Shalosh B. Ekhad And John E. MaJewicz \\ Submitted: February 17, 1995; Accepted: July 25, 1995 \\ Dedicated to Dominique Foata on the occasion of his sixtieth birthday.
}

AbstRACT. Using a certification procedure for Abel-type sums, we present a computerized proof of Abel's identity.

Abel's identity [1],[2] can be proved in many ways, including the elegant combinatorial methods of [3]. Here we present the first computer-generated proof using the methods introduced in [4].

Theorem([1]; [2], p.128). For $n \geq 0$ :

$$
\sum_{k=0}^{n}\left(\begin{array}{l}
n \\
k
\end{array}\right)(r+k)^{k-1}(s-k)^{n-k}=\frac{(r+s)^{n}}{r}
$$

Proof. Let $F_{n, k}(r, s)$ and $a_{n}(r, s)$ denote, respectively, the summand and sum on the LHS of (1), and let $G_{n, k}:=(s-n)\left(\begin{array}{c}n-1 \\ k-1\end{array}\right)(k+r)^{k-1}(s-k)^{n-k-1}$. Since

$F_{n, k}(r, s)-s F_{n-1, k}(r, s)-(n+r) F_{n-1, k}(r+1, s-1)+(n-1)(r+s) F_{n-2, k}(r+1, s-1)=G_{n, k}-G_{n, k+1}$,

(check!), we have by summing from $k=0$ to $k=n$, thanks to the telescoping on the right:

$$
a_{n}(r, s)-s a_{n-1}(r, s)-(n+r) a_{n-1}(r+1, s-1)+(n-1)(r+s) a_{n-2}(r+1, s-1)=0 .
$$

Since $(r+s)^{n} \cdot r^{-1}$ also satisfies this recurrence (check!) with the same initial conditions $a_{0}(r, s)=r^{-1}$ and $a_{1}(r, s)=(r+s) \cdot r^{-1},(1)$ follows.

We thank Herb Wilf for a great shrinking comment on an earlier (much longer) version.

\section{REFERENCES}

1. N. Abel, Beweis eines Ausdruckes, von welchem die Binomial-Formel ein einzelner Fall ist, Crelle's J. Mathematik (1826), 159-60.

2. Louis Comtet, Advanced Combinatorics, D. Reidel Publ. Co., Dordrecht/Boston, 1974, p. 128.

3. D. Foata, Enumerating k-Trees, Disc. Math. (1971), 181-186.

4. J. Majewicz, WZ-type certification procedures and Sister Celine's technique for Abel-type sums,, to appear (also available via anonymous ftp to ftp.math.temple.edu in directory pub/jmaj ).

Department of Mathematics, Temple University, Philadelphia, PA 19122

E-mail address: ekhad@math.temple.edu,jmaj@euclid.math.temple.edu 